\title{
Early postoperative MRI and detection of residual adenoma after transsphenoidal pituitary surgery
}

\author{
Lea M. Alhilali, MD, ${ }^{1}$ Andrew S. Little, MD, ${ }^{2}$ Kevin C. J. Yuen, MD, ${ }^{3}$ Jae Lee, MD, ${ }^{1}$ \\ Timothy K. Ho, MD, ${ }^{1}$ Saeed Fakhran, MD, ${ }^{4}$ and William L. White, MD ${ }^{2}$ \\ Departments of ${ }^{1}$ Neuroradiology, ${ }^{2}$ Neurosurgery, and ${ }^{3}$ Neurology and Barrow Neuroendocrinology Clinic, Barrow Neurological \\ Institute, St. Joseph's Hospital and Medical Center, Phoenix, Arizona; and ${ }^{4}$ Department of Radiology, Radiology Partners, \\ Banner Health and Hospital System, Phoenix, Arizona
}

\begin{abstract}
OBJECTIVE Current practice guidelines recommend delayed ( $\geq 3$ months after operation) postoperative MRI after transsphenoidal surgery for pituitary adenomas, although this practice defers obtaining important information, such as the presence of a residual adenoma, that might influence patient management during the perioperative period. In this study, the authors compared detection of residual adenomas by means of early postoperative (EPO) MRI (< 48 hours postsurgery) with both surgeon intraoperative assessment and late postoperative (LPO) MRI at 3 months.
\end{abstract}

METHODS Adult patients who underwent microscopic transsphenoidal surgery for pituitary adenomas with MRI preoperatively, $<48$ hours after the operation, 3 months postoperatively, and yearly for 4 years were included. The presence or absence of residual tumor was assessed intraoperatively by a single surgeon and postoperatively by 2 neuroradiologists blinded to the intraoperative assessment and other postoperative imaging studies. The presence of residual tumor was confirmed by reresection, tumor growth on imaging, or hormonal evidence. Interreader reliability was calculated at each imaging time point. Specificity, sensitivity, positive predictive value, and negative predictive value for EPO and LPO imaging and intraoperative assessment were determined.

RESULTS In total, 102 consecutive patients who underwent microscopic transsphenoidal resection of a pituitary adenoma were included. Eighteen patients (18\%) had confirmed residual tumors (12 confirmed by tumor growth, 5 by surgery, and 1 by biochemical evidence of persistent disease). Interreader reliability for detecting residual tumor on EPO MRI was almost perfect $(\kappa=0.88)$ and significantly higher than that for LPO MRI $(\kappa=0.69, p=0.03)$. EPO MRI was highly specific for residual tumor (98\%), a finding similar to that for intraoperative assessment $(99 \%, p=0.60)$ and significantly higher than that for LPO MRI $(81 \%, p<0.001)$. Notably, EPO MRI was significantly more sensitive for residual tumor $(100 \%)$ than both intraoperative assessment $(78 \%, p=0.04)$ and LPO MRI $(78 \%, p=0.04)$. EPO MRI had a 100\% negative predictive value and was used to find 4 residual tumors that were not identified intraoperatively. Residual tumors found on EPO MRI allowed for reresection during the same hospitalization for 3 patients.

CONCLUSIONS EPO MRI after transsphenoidal pituitary surgery can be reliably interpreted and has greater sensitivity for detecting residual tumor than intraoperative assessment and LPO MRI. This result challenges current guidelines stating that delayed postoperative imaging is preferable to early imaging. Pituitary surgeons should consider performing EPO MRI either in addition to or instead of delayed imaging.

https://thejns.org/doi/abs/10.3171/2019.11.JNS191845

KEYWORDS magnetic resonance imaging; pituitary adenoma; transsphenoidal; pituitary surgery

$\mathrm{D}$ ESPITE improvements in medical and radiation treatment, surgery remains the primary treatment for symptomatic non-prolactin-secreting adenomas and other sellar lesions. ${ }^{7}$ Although a total excision is often attempted, gross-total resection is achieved in only
$50 \%$ to $80 \%$ of pituitary macroadenomas. ${ }^{5,7,20}$ Detecting residual tumor in the early postoperative period is important because early reoperation can be performed using the same transsphenoidal approach before the development of adhesions. ${ }^{38}$ While the surgeon may attempt to assess for

ABBREVIATIONS EPO = early postoperative (< 48 hours postsurgery); iMRI = intraoperative MRI; LPO = late postoperative ( 3 months postsurgery) SUBMITTED July 3, 2019. ACCEPTED November 11, 2019.

INCLUDE WHEN CITING Published online February 7, 2020; DOI: 10.3171/2019.11.JNS191845. 
residual tumor intraoperatively, this method is highly subjective and limited..$^{16,19,28,37}$ Previous studies have suggested intraoperative assessments may miss residual tumors in nearly $20 \%$ of cases. ${ }^{10}$

Endocrinologic evaluation may be helpful for detecting residual tumors in patients with hormonally active tumors, but this method fails for nonfunctioning lesions, which constitute the majority of surgical lesions..$^{14}$ Even in functional tumors, early biochemical results may not accurately reflect surgical success or failure., ${ }^{1,411,26}$

MRI is the standard of care for evaluation of sellar lesions $;{ }^{6}$ however, its use in the immediate postoperative period is controversial, with several studies suggesting that early postoperative (EPO) imaging cannot be reliably interpreted. ${ }^{2,17,18,21,31}$ As a result, the neurosurgical consensus guidelines for postoperative follow-up after transsphenoidal resection do not recommend EPO MRI. ${ }^{39}$ However, studies suggesting that EPO MRI is inaccurate have relied upon outdated MRI methods. ${ }^{2,17,18,21,31}$ More recent studies have suggested that EPO MRI is as accurate as late postoperative (LPO) MRI at 3 months. ${ }^{29,34}$ However, these studies have failed to compare both imaging tests against an independent gold standard.

At our institution, patients undergoing microscopic resection of adenomas receive both EPO ( $<48$ hours after surgery) and LPO (3 months after surgery) MRI. This protocol is based on our own experience, in which EPO MRI has demonstrated its value by providing actionable clinical information regarding residual tumor and the need for immediate reresection. Our protocol of performing both EPO and LPO MRI for patients with pituitary adenomas provides an opportunity to directly compare the value of EPO MRI with the current standard of surgical assessment followed by delayed imaging at 3 months after surgery. Therefore, we sought to determine whether EPO MRI outperforms surgical assessment and LPO MRI for detecting residual tumor after transsphenoidal resection using surgical, clinical, and follow-up evidence of residual disease as the gold standard.

\section{Methods}

\section{Patient Selection and Image Acquisition}

This study was approved by the institutional review board at St. Joseph's Hospital and Medical Center (Phoenix, Arizona), with a waiver of informed consent. The study adhered to the US Code of Federal Regulations, Title 45, Part 46, Protection of Human Subjects (revised January 15, 2009).

We evaluated sequential patients between January 1, 2013, and December 31, 2014, who underwent transsphenoidal resection of a pituitary lesion at our institution, performed by a single neurosurgeon with 40 years of experience in microscopic transsphenoidal surgery (W.L.W.). Inclusion criteria were age greater than 18 years, contrastenhanced MRI performed less than 48 hours after surgery (EPO MRI), contrast-enhanced MRI performed 3 months after surgery (LPO MRI), follow-up of at least 5 years, and monitoring for the presence of residual tumor with imaging and biochemical testing. Patients were excluded from the analysis if they had no lesion identi- fied on MRI (Cushing disease) (3 patients), unavailable operative report (10 patients), lack of a direct statement or definitive language in the operative report as to whether a gross-total resection was achieved (5 patients), or motion degradation of MRI that precluded interpretation (0 patients).

The gold standard for the presence of residual tumor was either a pathologic specimen from immediate reresection, findings of growth on follow-up imaging, biochemical evidence of residual tumor, or a pathologic specimen from remote reresection. Patient demographic characteristics and clinical information were abstracted from the medical records.

All patients received contrast-enhanced MRI scans less than 48 hours after surgery and 3 months after surgery. MRI was obtained with the use of 1.5- or 3-T systems (Signa, GE Healthcare) with a standard head coil as follows: pre- and postcontrast sagittal and coronal T1-weighted imaging (repetition time [msec]/echo time [msec], 600/minimum setting; section thickness, $3 \mathrm{~mm}$; matrix, $288 \times 192$; number of acquisitions, 1-2); coronal T2-weighted imaging (6000/90; section thickness, 3 mm; matrix, $288 \times 192$; number of acquisitions, 3); axial FLAIR imaging (9000-10,000/149; inversion time, 2200 $\mathrm{msec}$ ); axial diffusion-weighted imaging (single-shot echo planar imaging; 10,000/minimum setting; section thickness, $5 \mathrm{~mm}$; matrix, $128 \times 256$ ); and axial T2-weighted imaging (6000/90; number of acquisitions, 1.5; section thickness, $5 \mathrm{~mm}$ ). Postcontrast imaging with a 3D axial spoiled gradient recalled acquisition in steady state was also performed (6.8/2.2; inversion time, $450 \mathrm{msec}$; flip angle, $12^{\circ}$; section thickness, $1 \mathrm{~mm}$; number of acquisitions, 1). Postcontrast imaging was performed immediately after the administration of $0.1 \mathrm{mmol} / \mathrm{kg}$ gadolinium contrast material (MultiHance, Bracco Diagnostics).

A fellowship-trained neuroradiologist (L.M.A.) used semiautomated segmentation of preoperative MRI scans (3D Slicer, version 4.8, http://www.slicer.org) to measure preoperative tumor volumes and the resection cavity size on both the EPO and LPO MRI scans. The same neuroradiologist measured the distance between the resection cavity and the chiasm by using the linear measurement tool in the PACS. Knosp grade 3 and 4 lesions were considered to invade the cavernous sinus. ${ }^{23,25}$

\section{Image Interpretation}

Two fellowship-trained neuroradiologists (L.M.A. and J.L.) independently reviewed the EPO MR images in randomized order for the presence or absence of residual tumor. The neuroradiologists were allowed to view the preoperative MR images but were blinded to all clinical data. The same radiologists subsequently assessed all LPO MR images for the presence or absence of residual tumor in a separate session, during which they were able to reference only the preoperative study and not the EPO MRI and were blinded to all clinical findings, including the intraoperative and EPO MRI findings. The studies were finally reviewed in a third session by both neuroradiologists, and a consensus opinion as to the presence or absence of residual tumor was obtained for sensitivity and specificity analysis. All 3 of the interpretation sessions were each 
TABLE 1. Demographic and clinical characteristics of patients who underwent microscopic transsphenoidal resection of a pituitary adenoma

\begin{tabular}{lc}
\hline \multicolumn{1}{c}{ Characteristic } & Value \\
\hline Total no. of patients & 102 \\
\hline Age at surgery, yrs & $50.7 \pm 15.4$ \\
\hline Male sex & $55(54)$ \\
\hline Type of pituitary lesion & \\
\hline Nonfunctioning adenoma & $66(65)$ \\
\hline ACTH-positive adenoma & $17(17)$ \\
\hline GH-positive adenoma & $11(11)$ \\
\hline Prolactinoma & $8(8)$ \\
\hline Macroadenoma & $93(91)$ \\
\hline Lesion vol, ml & $5.9 \pm 7.6$ \\
\hline Cavernous sinus invasion & $7(7)$ \\
\hline Chiasmatic compression & $58(57)$ \\
\hline Visual symptoms & $56(55)$ \\
\hline
\end{tabular}

$\mathrm{ACTH}=$ adrenocorticotropic hormone; $\mathrm{GH}=$ growth hormone.

Values are presented as number (\%) of patients unless otherwise indicated.

Mean values are presented \pm SD

separated by at least 2 weeks, and studies were viewed in a randomized order to minimize memory bias.

\section{Data Analysis}

Means and standard deviations were calculated for the descriptive data. Confidence intervals (CIs) for proportions were used with a continuity correction. ${ }^{27}$ Comparison of magnet strengths was performed with a chi-square test. A comparison of demographic and clinical data between patients with gross-total and subtotal resections was performed with an unpaired t-test for continuous variables and a Fisher exact test for categorical variables.

Interreader reproducibility for the presence or absence of residual tumor was compared for EPO and LPO MRI using Cohen's kappa coefficient and interpreted as follows: no agreement $(\leq 0)$, none to slight $(0.01-0.20)$, fair $(0.21-$ $0.40)$, moderate $(0.41-0.60)$, substantial (0.61-0.80), and almost perfect agreement $(0.81-1.00){ }^{22}$ Given the sample size, comparison of the kappa statistics was performed with a 2-sample z-test, as kappa is asymptotically normal.

The sensitivity and specificity for residual tumor were calculated with a confusion matrix for intraoperative assessment as well as the consensus interpretation of EPO and LPO MRI. Comparison among sensitivity and specificity values for the intraoperative assessment and MRI studies was performed with McNemar's chi-square test. ${ }^{12}$ A $p$ value of $<0.05$ was considered statistically significant.

\section{Results}

\section{Patient Selection and Image Acquisition}

The study included 102 patients (55 male, 47 female) who met the inclusion criteria. Demographic and clinical data are shown in Table 1. The sellar reconstruction included an autologous subcutaneous fat graft in nearly two-
TABLE 2. Comparison of demographic and clinical characteristics of patients who underwent microscopic transsphenoidal resection of a pituitary adenoma with gross-total or subtotal resection

\begin{tabular}{lccl}
\hline \multicolumn{1}{c}{ Characteristic } & $\begin{array}{c}\text { Gross-Total } \\
\text { Resection }(n=84)\end{array}$ & $\begin{array}{c}\text { Subtotal } \\
\text { Resection }(n=18)\end{array}$ & $p$ Value \\
\hline Age, yrs & $50.6 \pm 15.8$ & $50.7 \pm 13.8$ & $>0.99$ \\
\hline Male sex & $45(54)$ & $10(56)$ & $>0.99$ \\
\hline Lesion vol, $\mathrm{ml}$ & $4.5 \pm 5.4$ & $12.3 \pm 12.3$ & $<0.001$ \\
\hline $\begin{array}{c}\text { Cavernous sinus } \\
\text { invasion }\end{array}$ & $1(1)$ & $6(33)$ & $<0.001$ \\
\hline $\begin{array}{c}\text { Chiasmatic } \\
\text { compression }\end{array}$ & $44(52)$ & $14(78)$ & 0.07 \\
\hline
\end{tabular}

Data are presented as number (\%) of patients unless otherwise indicated. Mean values are presented \pm SD.

thirds of patients $(66 / 102,66 \%)$. There was no significant difference in the proportion of EPO MRI (34/102; 33\%) and LPO MRI $(27 / 102 ; 26 \%)$ performed on a 3-T system $(\mathrm{p}=0.28)$. Five patients had an asymptomatic hematoma on the EPO MRI $(5 / 102,5 \%)$.

Eighteen patients had confirmed residual tumors $(18 / 102 ; 18 \%)$. One patient with acromegaly had biochemical evidence of residual tumor without reresection or growth on MRI; in the remaining patients, the presence of residual tumors was determined by either reresection (n $=5$ ) or growth on subsequent imaging $(n=12)$. Patients with subtotal resections had significantly larger lesions and were more likely to have cavernous sinus invasion than patients with gross-total resection (both $\mathrm{p}<0.001$ ). Demographic and clinical features of those with grosstotal resection and subtotal resection are shown in Table 2.

Of 18 patients with subtotal resections, 12 received subsequent radiation, and of these, 11 underwent CyberKnife stereotactic radiosurgery. Eight of the 18 patients with residual tumors required reresection, and among these 8 patients, 6 reresections were performed within 6 months. Three of the 8 patients had reresection performed during the same hospitalization after EPO MRI confirmed the presence of a residual tumor that the surgeon considered potentially resectable. Two patients with prolactinomas received a subtotal resection; however, neither of these patients received medical therapy between the EPO and LPO scans, as medical therapy for prolactinomas is not invariably restarted after incomplete resection at our institution, especially if the patient is likely to undergo radiation treatment.

The mean EPO MRI cavity volume was $3.3 \mathrm{ml}$ (range 0.1-16.3 ml), compared with a mean volume of $1.6 \mathrm{ml}$ on the LPO MRI (range 0-8.7 ml). Overall, the mean percentage decrease in cavity volume from the EPO MRI to the LPO MRI was $51.5 \%$. This resulted in a significant change in the mean distance between residual sellar tissue and chiasm between the 2 scans, with a mean distance of $2.5 \mathrm{~mm}$ on the EPO and $4.7 \mathrm{~mm}$ on the LPO MRI scan. The percentage change in craniocaudal dimension of resection cavities from EPO to LPO scans was higher for 

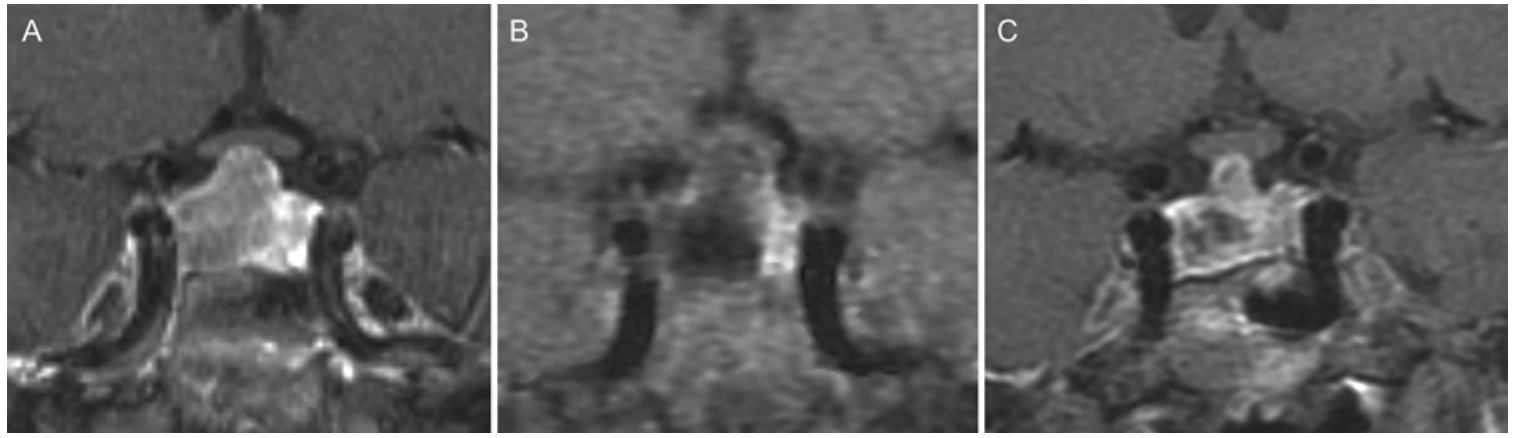

FIG. 1. Enhancing postoperative material on LPO MRI (3 months after surgery). T1-weighted, fat-saturation, postcontrast coronal images in a representative patient. A pituitary macroadenoma is identified on initial preoperative imaging $(\mathbf{A})$ extending to the right lateral sella and with mild mass effect on the chiasm. The EPO MR image ( $<48$ hours after surgery) (B) demonstrates a grosstotal resection. The resection cavity on the EPO MR image demonstrates a shape similar to that of the preoperative lesion but lacks any enhancement, helping the radiologists to clearly identify removal of the entire lesion. The LPO MR image (C) demonstrates collapse of the resection cavity, which now displays some internal enhancement. This enhancement renders assessment for residual tumor more difficult in comparison with the EPO scan and may result in more inconsistent and inaccurate readings.

cavities without fat packing $(63.2 \%)$ than for cavities with fat packing $(52.1 \%)(\mathrm{p}=0.03)$. The mean time to recurrence was 2.8 years (range $1-5$ years).

\section{Image Interpretation}

The kappa statistic was used to measure interobserver agreement for the presence or absence of residual tumor on EPO MRI to determine whether EPO images can be reliably and reproducibly interpreted. The kappa statistic for the presence or absence of residual tumor for EPO MRI was 0.88 (95\% CI 0.77-1.0), indicating near-perfect agreement. This was significantly higher than the kappa statistic for the LPO MRI $(\kappa=0.69,95 \%$ CI $0.54-0.84$, $\mathrm{p}=0.03$ ), which demonstrated substantial agreement. Most of the disagreements between observers regarding the LPO MRI results were related to whether enhancing tissue in the operative bed represented residual tumor or granulation tissue; this was not an issue with the interpretation of EPO MRI (Fig. 1).

The sensitivity and specificity of EPO MRI, intraoperative assessment, and LPO MRI were compared (Table 3). EPO MRI had a high specificity for residual tumor (98\%, 95\% CI 92\%-100\%), which was similar to the specificity of the intraoperative assessment $(99 \%, 95 \%$ CI $94 \%-100 \%$, $p=0.60)$. EPO MRI had only 2 false-positive examination findings. The first false-positive finding was in a patient with a nonfunctioning adenoma who had a previous resection, in whom a region of previous postoperative change in the sella was mistaken for residual tumor on the EPO MRI. However, these postoperative changes were stable prior to the reresection and remained so over the follow-up time period of this study, confirming that the region represented postoperative change. The second false-positive finding was in a patient with a macroadenoma and acromegaly, in whom very nodular enhancement at the edge of the resection cavity was felt to represent residual tumor on the EPO MRI. However, this nodular enhancement had resolved on the 1-year follow-up MRI. A representative example of a false-positive interpretation on EPO MRI is shown in Fig. 2. Of note, the specificity of EPO MRI (98\%, 95\% CI 92\%-100\%) was significantly higher $(\mathrm{p}<0.001)$ than that of LPO MRI (81\%, 95\% CI 71\%-89\%).

Additionally, EPO MRI had significantly higher sensitivity for residual tumor $(100 \%, 95 \%$ CI $82 \%-100 \%)$ than both intraoperative assessment $(78 \%, 95 \%$ CI $52 \%-94 \%, p$ $=0.04)$ and LPO MRI (78\%, 95\% CI 52\%-94\%, p = 0.04). The high sensitivity of EPO MRI resulted in a $100 \%$ negative predictive value for this test.

TABLE 3. Sensitivity and specificity analysis for EPO MRI compared with intraoperative assessment and LPO MRI

\begin{tabular}{lccccc}
\hline & & & & \multicolumn{2}{c}{$p$ Value } \\
\cline { 5 - 6 } \multicolumn{1}{c}{ Statistic } & EPO MRI & Intraop & LPO MRI & EPO vs Intraop & EPO vs LPO \\
\hline Sensitivity & $100(82-100)$ & $78(52-94)$ & $78(52-94)$ & 0.04 & 0.04 \\
\hline Specificity & $98(92-100)$ & $99(94-100)$ & $81(71-89)$ & 0.60 & $<0.001$ \\
\hline Positive predictive value & $90(67-97)$ & $93(66-99)$ & $47(35-59)$ & 0.76 & $<0.001$ \\
\hline Negative predictive value & $100(\mathrm{NA})$ & $96(91-98)$ & $94(88-98)$ & 0.07 & 0.03 \\
\hline Accuracy & $98(93-100)$ & $96(90-99)$ & $80(71-88)$ & 0.40 & $<0.001$ \\
\hline
\end{tabular}

Intraop = intraoperative assessment; NA = not applicable.

Values are presented as percentage $(95 \% \mathrm{Cl})$ unless otherwise indicated. Boldface type indicates statistical significance. 

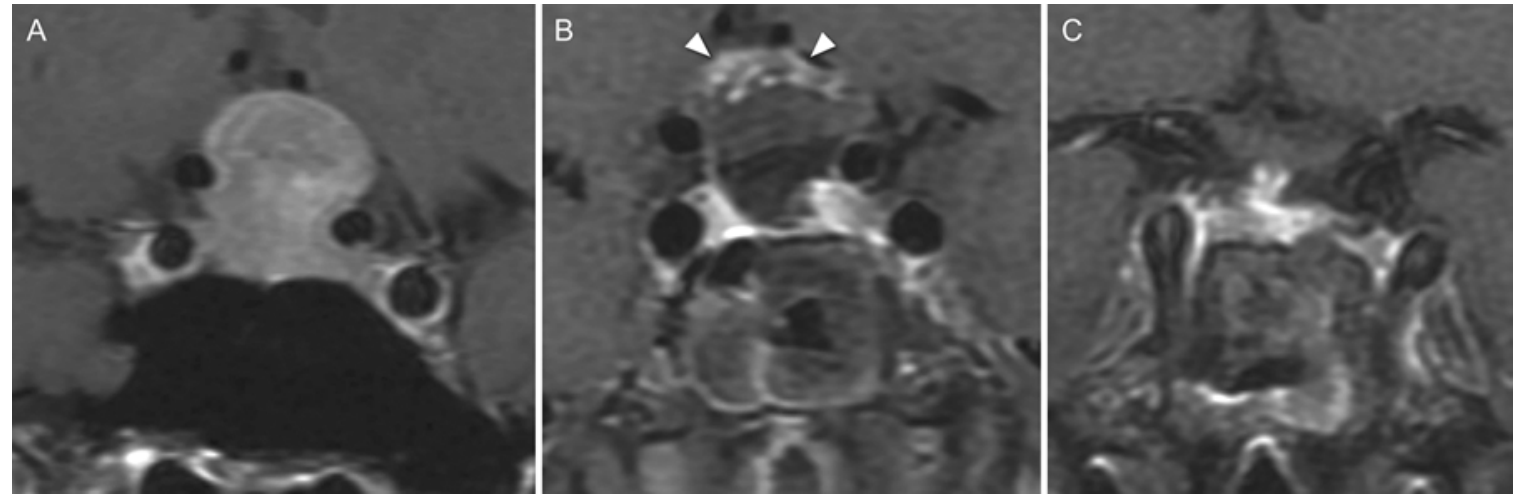

FIG. 2. False-positive EPO MRI (< 48 hours after surgery) findings. T1-weighted, fat-saturation, postcontrast coronal images in a patient with false-positive EPO MRI findings. A pituitary macroadenoma is identified on initial preoperative imaging $(\mathbf{A})$ with significant mass effect on the chiasm. On EPO MRI (B), enhancing material at the superior aspect of the resection cavity (white arrowheads) was believed to represent residual tumor. However, on long-term follow-up 3 years later (C), the abnormal soft tissue is no longer seen, consistent with evolving postoperative change.

The EPO MRI in 4 patients showed residual tumors that were not identified intraoperatively ( 4 of 18 residual tumors). Of these residual tumors not recognized at surgery, 2 were at the periphery of large macroadenomas and 2 were in patients with Cushing disease who required surgical reexploration, 1 within the sella and 1 with clivus invasion. In 1 patient with Cushing disease, the finding of a small residual tumor in the sella on the EPO MRI was accompanied by a high serum cortisol level (71 $\mu \mathrm{g} /$ dl) on the first postoperative morning, and the patient subsequently underwent immediate surgical reexploration. In the other patient with Cushing disease, the residual tumor invading the clivus was closely observed, and the patient had both imaging progression and biochemical recurrence that required surgical reexploration 4 years later. Figure 3 illustrates a lesion missed by the surgical assessment that was identified by EPO MRI yet was difficult to detect with LPO MRI.
LPO MRI did not enable correct categorization of those patients who were incorrectly categorized by using EPO MRI. Specifically, the 2 false-positive examination findings on EPO MRI were also incorrectly interpreted as positive for residual tumors on LPO MRI. Additionally, 18 patients who had been correctly categorized by EPO MRI were incorrectly categorized by LPO MRI. Fourteen patients with true-negative findings that were correctly classified by EPO MRI were incorrectly classified as having positive findings by LPO MRI. Patients with false-positive findings on LPO were more likely to have Gelfoam (Pfizer), DuraGen (Integra LifeSciences), or pedicled nasoseptal flaps used in the sellar reconstruction compared with the overall cohort $(69 \%$ vs $44 \%, p=0.049)$. Figure 4 provides 2 examples for which EPO MR images enabled correct identification of a gross-total resection, whereas LPO MR images of enhancing postoperative tissue in the cavity resulted in the incorrect diagnoses of residual tu-
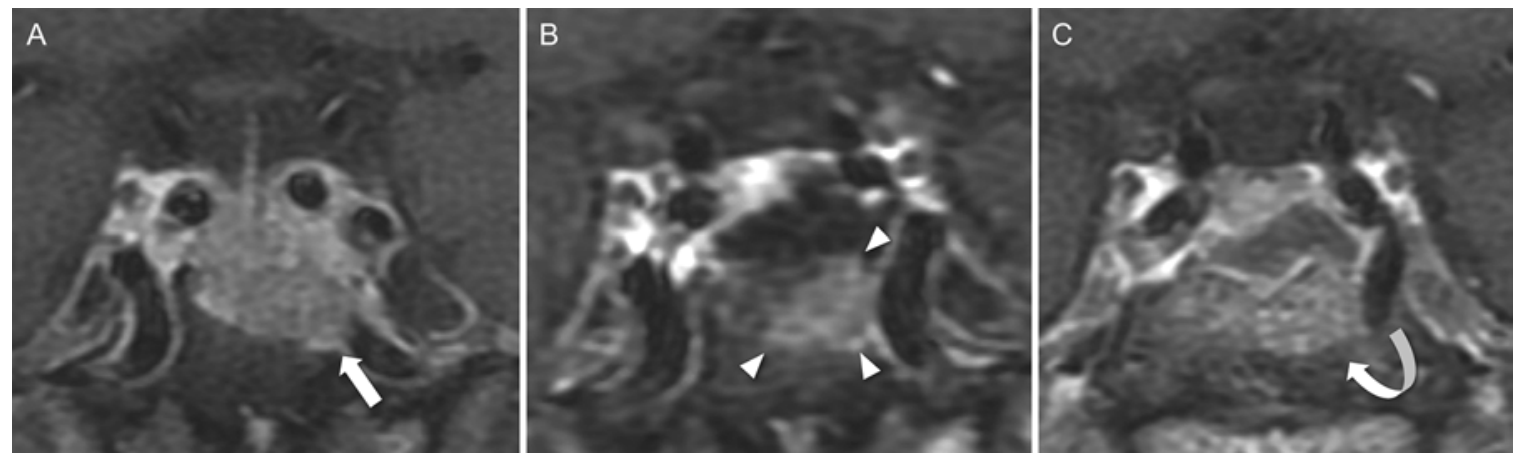

FIG. 3. False-negative surgical assessment detected with EPO MRI $(<48$ hours after surgery). T1-weighted, fat-saturation, postcontrast coronal images in a patient with a false-negative surgical assessment. A pituitary macroadenoma is identified on initial preoperative imaging (A) invading the clivus (white arrow). At the time of surgery, it was believed that a gross-total resection had been achieved. However, on the EPO MR image (B), residual tumor is identified within the clivus (white arrowheads) that had not been detected surgically. On the LPO MR image (3 months after surgery) (C), the residual enhancing material in the clivus is still present (curved arrow); however, it is significantly more difficult to detect, because the clivus itself now demonstrates enhancement from postoperative change, and enhancing material within the resection cavity makes it difficult to identify the outline of the residual tumor. 

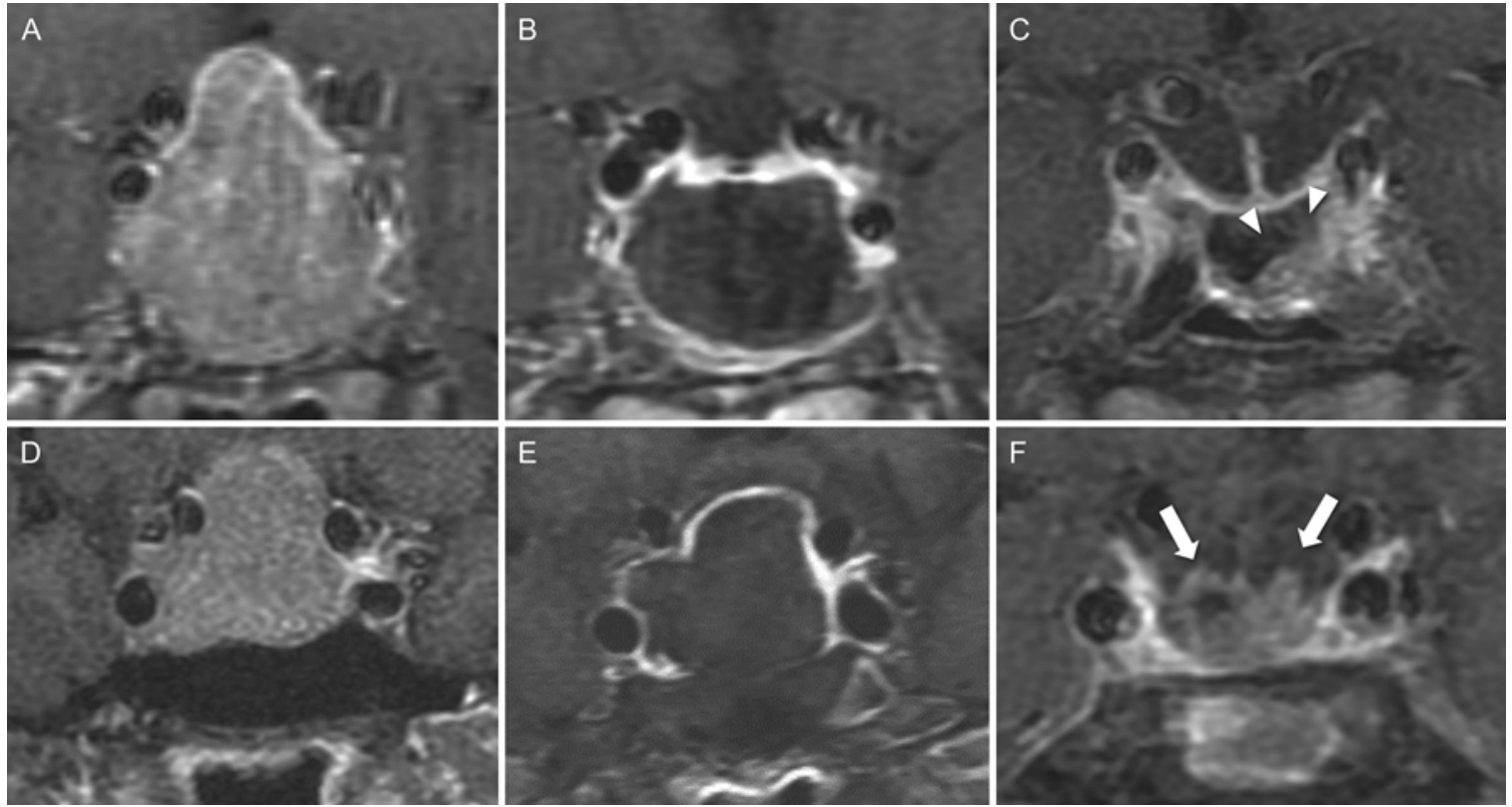

FIG. 4. False-positive LPO MRI (3 months after surgery) findings, correctly classified with the use of EPO MRI (< 48 hours after surgery). T1-weighted, fat-saturation, postcontrast, coronal images in 2 patients with false-positive LPO MRI findings, which were correctly classified with the use of EPO MRI. A pituitary macroadenoma can be identified on initial preoperative imaging (A and D) in the 2 patients. On EPO MR images for both patients (B and E), there is no enhancing soft tissue in the resection cavity, and gross-total resections were correctly diagnosed. However, the LPO MR images for both patients (C and F) show nodular enhancing material within the resection cavity (white arrowheads, C; white arrows, F) that was incorrectly classified as residual tumor and later resolved.

mor. Additionally, in 4 patients true-positive findings that were correctly classified with EPO MRI were incorrectly classified with LPO MRI as negative findings. Figure 5 presents an example of a residual tumor that was correctly classified with EPO MRI and missed with LPO MRI.

\section{Discussion}

It has been the practice of the senior surgeon at our in- stitution (W.L.W.) to perform EPO MRI on pituitary cases because of his impression that the scans provided clinically meaningful information. For example, he has found EPO MRI to contain actionable information, such as residual adenoma, that prevents the "surprise" of discovering residual adenoma 3 months after a patient has healed from surgery. However, this impression has not previously been subjected to rigorous evaluation. We undertook this study to evaluate the potential utility of EPO MRI, using mod-
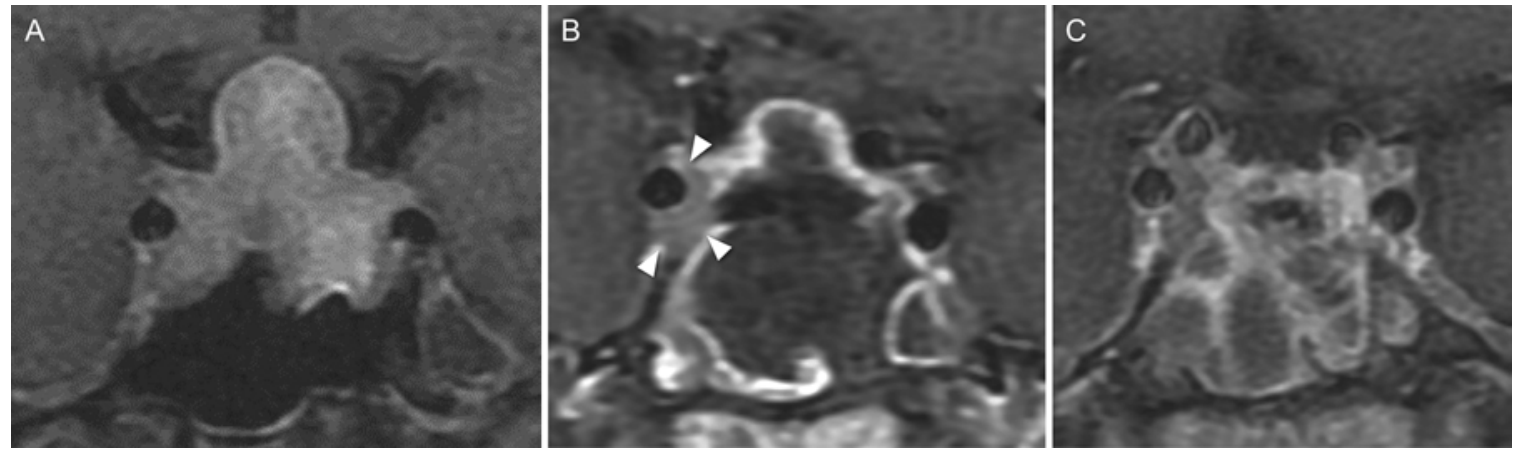

FIG. 5. False-negative LPO MRI findings (3 months after surgery), correctly classified with the use of EPO MRI (< 48 hours after surgery). T1-weighted, fat-saturation, postcontrast coronal images for a patient with false-negative LPO MRI findings, correctly classified with the use of EPO MRI. A pituitary macroadenoma is identified on initial preoperative imaging $(A)$ with prominent mass effect on the chiasm, extending toward the right cavernous sinus. On the EPO MR image (B), there is residual tumor in the region of the right cavernous sinus (white arrowheads) that was also recognized intraoperatively. However, on the LPO MR image (C), the extensive enhancing postoperative change makes it difficult to recognize this residual tumor in the cavernous sinus, obscuring the residual tumor as a result of the extensive background enhancement. 
ern imaging techniques evaluated by experienced neuroradiologists at a high-volume academic pituitary center. We discovered that, in patients undergoing transsphenoidal resection of a pituitary adenoma, EPO MRI had increased sensitivity for detecting residual tumor and was superior to LPO MRI in terms of both diagnostic performance and interreader reliability. Furthermore, EPO MRI outperformed surgeon intraoperative assessment of extent of resection. In addition, our study suggests that, among patients with transsphenoidal pituitary resection in whom EPO MRI has been performed, LPO MRI does not result in detection of any additional residual tumor, and in fact EPO MRI may be preferable to delayed MRI.

As shown in the case examples (Figs. 1-5), interpreting LPO MR images is challenging even for experienced surgeons and radiologists. These examples highlight the changes in sellar anatomy and the emergence of nonspecific areas of postoperative enhancement that occur in the resection cavity at 3 months. The increasing use of Gelfoam in transsphenoidal resections has only increased the prevalence of confounding enhancement on LPO MRI, as Gelfoam incites contrast-enhancing granulation tissue as it degrades. This enhancing granulation tissue is not visible until 3 months after surgery and can lead to an incorrect diagnosis of residual tumor or overestimation of the amount of residual tumor with LPO MRI. ${ }^{15}$ Other materials, such as DuraGen and vascularized flaps, can also show enhancement that varies over time, which is most prominent and most confounding at the time of the LPO MRI. ${ }^{3}$ In fact, the majority of patients with false-positive LPO MRI findings had sellar reconstruction using these implanted materials or flaps. As endoscopic approaches become increasingly popular, ${ }^{19}$ use of these implanted materials or flaps will become increasingly common, and the likelihood of false-positive findings will increase if postoperative imaging is delayed to 3 months. Additionally, compared with EPO MRI, LPO MRI shows distorted sellar anatomy related to granulation tissue formation and inflammatory changes that can shift normal anatomical structures. This makes direct comparison to preoperative imaging more difficult on LPO MRI than EPO MRI, further complicating the search for residual tumor. ${ }^{15}$

The current recommendation that EPO MRI not be routinely performed after transsphenoidal resection is predominantly based on studies that were published more than 15 years ago. ${ }^{17,18,31}$ However, the imaging methods used in those studies do not reach the current standards for sellar imaging. The techniques used in those studies lack the basic standards now seen in pituitary MRI, such as fatsaturation imaging, high-resolution coronal T2-weighted imaging, and volumetric imaging. Even the parameters of the cornerstone sagittal and coronal T1-weighted images in these studies do not meet the current standard of care, with larger fields of view and slice thickness than currently recommended..$^{9}$ In a more recent study, on which the recommendation to forego EPO MRI was based, intraoperative MRI (iMRI), rather than true EPO MRI, was used for evaluation of residual tumor. ${ }^{2}$ These authors found there was not significant shrinkage in the resection cavity on the last intraoperative scan. However, to avoid contrast leakage through tumor remnants because the images were obtained intraoperatively, contrast medium was not used in most patients. Therefore, this study should not be considered a true evaluation of early postoperative imaging, as the intraoperative technique and lack of contrast do not reflect the protocol used in true EPO MRI.

More recent studies using modern MR techniques that are now the standard of care in pituitary imaging have indicated that EPO MRI is very accurate, with efficacy similar to that of LPO MRI at 3 months..$^{29,34}$ However, these studies used LPO MRI as the gold standard by which EPO MRI was judged, and neither study addressed the consideration that EPO MRI may, in fact, be more accurate than LPO MRI. As a result, neither study used an external gold standard, such as reresection or long-term follow-up, to determine whether LPO MRI findings were accurate. Our study, to our knowledge, is the first to demonstrate that EPO MRI is not only accurate in determining residual tumor but also more accurate than LPO MRI, with superior interreader reliability. Furthermore, we found that LPO MRI detected no additional lesions in the setting of prior EPO MRI. In fact, discrepancies between LPO and EPO MRI were all settled in favor of EPO MRI. This result suggests that EPO MRI rather than LPO MRI should be used as the primary postoperative follow-up study in patients undergoing transsphenoidal pituitary surgery. After the initial EPO MRI, our data support the current recommendations of yearly surveillance, because the mean time to recurrence was 2.8 years in our cohort.

In addition to being more accurate for detecting residual tumor, EPO MRI provides other benefits. First, it provides the surgeon with an immediate "educational" opportunity as to whether a complete excision was achieved or whether the size of the inserted Gelfoam was adequate. Delaying imaging until 3 months may make it difficult for the surgeon to recollect what was observed during the surgical procedure. Similarly, because EPO MRI is performed in the immediate postoperative period, it can help guide or influence immediate reresection. By contrast, if reresection is delayed until residual tumor is detected on an LPO MRI, the surgery may be technically more difficult because of scar tissue or postoperative fibrosis that may develop in the 3-month postoperative interval. Furthermore, given the high negative predictive value of EPO MRI, a negative scan can be used to effectively determine if a patient is clear of residual tumor and may encourage less frequent follow-up imaging, which translates into a cost-saving exercise. Finally, as the imaging is performed during the same hospitalization, there is no risk that the patient will be lost to follow-up, which may occur if imaging is delayed by 3 months and performed in an outpatient setting. In fact, in prior studies, nearly half of the initial cohort of surgical patients did not undergo their 3-month follow-up imaging. ${ }^{34}$ Another advantage for the patient of performing the imaging during the same hospitalization is that the imaging can be included in the bundled hospital payment and will not incur an additional charge to the patient, as it would in the outpatient setting. ${ }^{36}$

An alternative to EPO MRI that has been advocated by some authors is iMRI, performed with either a highor low-field technique. ${ }^{8,30}$ Studies have shown that iMRI may identify residual tumor missed by the surgeon and 
increase the likelihood of gross-total resection. ${ }^{30}$ However, installation of an iMRI suite is expensive, requiring a substantial investment in time and money to construct and remodel the operating theater. ${ }^{8}$ In addition, iMRI increases the duration of the index surgery. This limits implementation of this technology to all but the largest centers and does not provide a general substitute for early postoperative imaging. Additionally, tumor may be mistaken for hematoma on iMRI, meaning that iMRI does not have the same strong negative predicative value as EPO MRI and therefore may not prove to be as beneficial. ${ }^{33}$

Although the EPO MRI outperformed the LPO MRI for detection of residual tumor, there are situations in which the LPO MRI may provide important diagnostic information for specific patients. After surgical resection, select patients will require adjuvant radiation therapy. There are several radiation modalities available for treatment. The choice of radiation modality is significantly influenced by the proximity of the tumor to local critical anatomy, namely the optic chiasm. ${ }^{24}$ The resection cavity is often similar in size to the original tumor in the early postoperative period and subsequently collapses by the time the delayed MRI is obtained at 3 months. ${ }^{31}$ In our study, we found patients to have, on average, an approximately 50\% reduction in the resection cavity size on the LPO MRI, with nearly double the distance between the resection cavity and the chiasm from the EPO MRI. Therefore, for a patient in whom residual tumor is detected on the EPO MRI and in whom radiation therapy is considered, an LPO may be helpful for treatment planning after the cavity has moved away from the chiasm. The collapse of the resection cavity on the LPO scan was less among patients who underwent packing of the cavity with endogenous fat than among those without fat packing. Therefore, if radiation therapy is planned for patients with fat packing, it may be beneficial to obtain imaging even later than 3 months, to allow further collapse of the cavity. Additionally, among patients who receive medical therapy for residual prolactinoma, it may be prudent to reimage and reassess residual tumor before deciding on definitive treatment.

A concern exists that EPO MRI will detect asymptomatic hematomas, thus causing unnecessary anxiety and follow-up among postoperative patients. A previous study estimated the incidence of asymptomatic hematoma to be as high as $16.5 \%$; however, in this study fat-suppressed imaging was not used, and therefore a portion of what was presumed to be hematoma may have been partially related to fat grafting. ${ }^{38}$ In our cohort, the rate of asymptomatic hematoma was much lower (5\%), and there was no significant difference in the degree of resection cavity collapse between patients with and those without asymptomatic hematoma findings on EPO MRI. Therefore, detection of asymptomatic hematoma will likely be a rare occurrence and can be managed expectantly, without having an effect on any delayed radiation treatment planning study.

\section{Study Limitations}

Our study had several limitations, including its retrospective design and use of patients treated by a single neurosurgeon. However, it is likely that the extensive experience of the neurosurgeon who performed the procedures in this study contributed to increased accuracy of the intraoperative assessment compared with the accuracy of less-experienced surgeons. This possibility suggests that using EPO MRI may be even more important with a lessexperienced surgeon. Additionally, our cohort consisted of patients with both functional and nonfunctional lesions. Arguably, MRI has its greatest benefit in treating patients with nonfunctional lesions, for which biochemical evaluation for residual tumors is not available, and prior studies have restricted patient populations to only those with nonfunctional lesions. ${ }^{17,18}$ However, given the very strong negative predictive value of EPO MRI, we believe that this procedure may also be helpful in treating patients with functional lesions, for which immediate biochemical findings may not be definitive in terms of establishing a complete resection. Furthermore, we did not have a representative population of aggressive tumors in our cohort, such as Crooke's cell adenomas or plurihormonal PIT-1-positive adenomas. Therefore, it would be difficult to make recommendations regarding biologically aggressive lesions on the basis of our study. However, it could be argued that among patients with biologically concerning lesions, detection of residual tumor in the early postoperative period is even more important, because immediate reresection is associated with less morbidity and may help decrease the tumor volume to be irradiated. Finally, the follow-up period for demonstrating residual tumor growth was relatively short; however, to ensure that EPO MRI was performed with current imaging techniques, we excluded data from EPO MRI studies older than 5 years. We believe that the 5 -year follow-up period is sufficient, because most growth of residual tumors and recurrences take place within 5 years. ${ }^{32}$ In fact, with a mean follow-up of 5 years, $95 \%$ of nonfunctioning adenomas will have some sort of progression. ${ }^{35}$ Furthermore, we increased the sensitivity for subtle tumor growth significantly with the use of $3 \mathrm{D}$ sequences and volumetric measurements. In fact, if nonfunctioning adenomas are followed with volumetric measurements and $3 \mathrm{D}$ sequences, a mean growth of $0.3 \mathrm{ml}$ per year can be detected. ${ }^{13}$ Such a small change may go unnoticed with a simple subjective interpretation by a radiologist. However, our automated segmentation and volumetric imaging process was specifically performed to detect these subtle changes, increasing the sensitivity for detection of tumor growth. Therefore, we believe that there is low risk that we would not detect change in residual tumor. However, it is possible that some tumor remnants were not characterized as such if their degree of growth remained below the threshold of our highly sensitive methods.

\section{Conclusions}

EPO MRI is accurate and reliable for the detection of residual tumor after transsphenoidal resection, increasing sensitivity for detecting residual tumors in the immediate postoperative period. Replacement of LPO MRI at 3 months with EPO MRI as the primary imaging followup method within the first postoperative year may not adversely impact detection of residual tumor and, in fact, may result in greater diagnostic accuracy and interreader reliability as well as earlier diagnosis and surgical treatment 
of residual disease. This study challenges current practice guidelines that favor delayed over early postoperative imaging, instead suggesting that EPO MRI can complement or possibly even replace delayed imaging among patients with pituitary adenomas.

\section{Acknowledgments}

We thank Heidi Jahnke, RN, MSN, for assistance in identifying patients as well as Corbin Stephens and Samuel Helms-Galbraith for assistance in collecting clinical information. We thank the staff of Neuroscience Publications at Barrow Neurological Institute for assistance with manuscript preparation.

\section{References}

1. Antunes X, Ventura N, Camilo GB, Wildemberg LE, Guasti A, Pereira PJM, et al: Predictors of surgical outcome and early criteria of remission in acromegaly. Endocrine 60:415422, 2018

2. Berkmann S, Schlaffer S, Buchfelder M: Tumor shrinkage after transsphenoidal surgery for nonfunctioning pituitary adenoma. J Neurosurg 119:1447-1452, 2013

3. Bladowska J, Bednarek-Tupikowska G, Sokolska V, Badowski $\mathrm{R}$, Moroń K, Bonicki W, et al: MRI image characteristics of materials implanted at sellar region after transsphenoidal resection of pituitary tumours. Pol J Radiol 75:46-54, 2010

4. Blevins LS Jr, Sanai N, Kunwar S, Devin JK: An approach to the management of patients with residual Cushing's disease. J Neurooncol 94:313-319, 2009

5. Buchfelder M: Treatment of pituitary tumors: surgery. Endocrine 28:67-75, 2005

6. Buchfelder M, Schlaffer S: Imaging of pituitary pathology. Handb Clin Neurol 124:151-166, 2014

7. Buchfelder M, Schlaffer S: Surgical treatment of pituitary tumours. Best Pract Res Clin Endocrinol Metab 23:677-692, 2009

8. Buchfelder M, Schlaffer SM: Intraoperative magnetic resonance imaging during surgery for pituitary adenomas: pros and cons. Endocrine 42:483-495, 2012

9. Chowdhury IN, Sinaii N, Oldfield EH, Patronas N, Nieman LK: A change in pituitary magnetic resonance imaging protocol detects ACTH-secreting tumours in patients with previously negative results. Clin Endocrinol (Oxf) 72:502-506, 2010

10. Dort JC, Sutherland GR: Intraoperative magnetic resonance imaging for skull base surgery. Laryngoscope 111:15701575,2001

11. Giustina A, Chanson P, Bronstein MD, Klibanski A, Lamberts S, Casanueva FF, et al: A consensus on criteria for cure of acromegaly. J Clin Endocrinol Metab 95:3141-3148, 2010

12. Hawass NE: Comparing the sensitivities and specificities of two diagnostic procedures performed on the same group of patients. Br J Radiol 70:360-366, 1997

13. Hwang K, Kwon T, Park J, Joo JD, Han JH, Oh CW, et al: Growth pattern and prognostic factors of untreated nonfunctioning pituitary adenomas. J Korean Neurosurg Soc 62:256-262, 2019

14. Karamouzis I, Caputo M, Mele C, Nuzzo A, Zavattaro M, Car P, et al: Transsphenoidal surgery for pituitary adenomas: early results from a single center. Hormones (Athens) 17:551-556, 2018

15. Kiliç T, Ekinci G, Seker A, Elmaci I, Erzen C, Pamir MN: Determining optimal MRI follow-up after transsphenoidal surgery for pituitary adenoma: scan at 24 hours postsurgery provides reliable information. Acta Neurochir (Wien) 143:1103-1126, 2001

16. Klopfenstein JD, Spetzler RF, Kim LJ, Feiz-Erfan I, Han PP,
Zabramski JM, et al: Comparison of routine and selective use of intraoperative angiography during aneurysm surgery: a prospective assessment. J Neurosurg 100:230-235, 2004

17. Kremer P, Forsting M, Hamer J, Sartor K: MR imaging of residual tumor tissue after transsphenoidal surgery of hormone-inactive pituitary macroadenomas: a prospective study. Acta Neurochir Suppl 65:27-30, 1996

18. Kremer P, Forsting M, Ranaei G, Wüster C, Hamer J, Sartor $\mathrm{K}$, et al: Magnetic resonance imaging after transsphenoidal surgery of clinically non-functional pituitary macroadenomas and its impact on detecting residual adenoma. Acta Neurochir (Wien) 144:433-443, 2002

19. Little AS, Chicoine MR, Kelly DF, Sarris CE, Mooney MA, White WL, et al: Evaluation of surgical resection goal and its relationship to extent of resection and patient outcomes in a multicenter prospective study of patients with surgically treated nonfunctioning pituitary adenomas: a case series. Oper Neurosurg (Hagerstown) 18:26-33, 2020

20. Little AS, Kelly DF, White WL, Gardner PA, FernandezMiranda JC, Chicoine MR, et al: Results of a prospective multicenter controlled study comparing surgical outcomes of microscopic versus fully endoscopic transsphenoidal surgery for nonfunctioning pituitary adenomas: the Transsphenoidal Extent of Resection (TRANSSPHER) Study. J Neurosurg [epub ahead of print March 22, 2019; DOI: 10.3171/2018.11. JNS181238]

21. Mansouri A, Symons S, Schwartz M, Chen J, Pirouzmand F: Quantitative volumetric analysis post transsphenoidal pituitary adenoma surgery. Can J Neurol Sci 39:600-604, 2012

22. McHugh ML: Interrater reliability: the kappa statistic. Biochem Med (Zagreb) 22:276-282, 2012

23. Micko AS, Wöhrer A, Wolfsberger S, Knosp E: Invasion of the cavernous sinus space in pituitary adenomas: endoscopic verification and its correlation with an MRI-based classification. J Neurosurg 122:803-811, 2015

24. Minniti G, Osti MF, Niyazi M: Target delineation and optimal radiosurgical dose for pituitary tumors. Radiat Oncol 11:135, 2016

25. Mooney MA, Hardesty DA, Sheehy JP, Bird R, Chapple K, White WL, et al: Interrater and intrarater reliability of the Knosp scale for pituitary adenoma grading. J Neurosurg 126:1714-1719, 2017

26. Netuka D, Masopust V, Belšán T, Kramář F, Beneš V: One year experience with 3.0 T intraoperative MRI in pituitary surgery. Acta Neurochir Suppl 109:157-159, 2011

27. Newcombe RG: Two-sided confidence intervals for the single proportion: comparison of seven methods. Stat Med 17:857872,1998

28. Orringer D, Lau D, Khatri S, Zamora-Berridi GJ, Zhang K, $\mathrm{Wu} \mathrm{C}$, et al: Extent of resection in patients with glioblastoma: limiting factors, perception of resectability, and effect on survival. J Neurosurg 117:851-859, 2012

29. Patel KS, Kazam J, Tsiouris AJ, Anand VK, Schwartz TH: Utility of early postoperative high-resolution volumetric magnetic resonance imaging after transsphenoidal pituitary tumor surgery. World Neurosurg 82:777-780, 2014

30. Patel KS, Yao Y, Wang R, Carter BS, Chen CC: Intraoperative magnetic resonance imaging assessment of non-functioning pituitary adenomas during transsphenoidal surgery. Pituitary 19:222-231, 2016

31. Rajaraman V, Schulder M: Postoperative MRI appearance after transsphenoidal pituitary tumor resection. Surg Neurol 52:592-599, 1999

32. Roelfsema F, Biermasz NR, Pereira AM: Clinical factors involved in the recurrence of pituitary adenomas after surgical remission: a structured review and meta-analysis. Pituitary 15:71-83, 2012

33. Schwartz TH, Stieg PE, Anand VK: Endoscopic transsphenoidal pituitary surgery with intraoperative magnetic reso- 
nance imaging. Neurosurgery 58 (1 Suppl):ONS44-ONS51, 2006

34. Stofko DL, Nickles T, Sun H, Dehdashti AR: The value of immediate postoperative MR imaging following endoscopic endonasal pituitary surgery. Acta Neurochir (Wien) 156:133-140, 2014

35. Tanaka Y, Hongo K, Tada T, Sakai K, Kakizawa Y, Kobayashi S: Growth pattern and rate in residual nonfunctioning pituitary adenomas: correlations among tumor volume doubling time, patient age, and MIB-1 index. J Neurosurg 98:359-365, 2003

36. Tompkins CP, Altman SH, Eilat E: The precarious pricing system for hospital services. Health Aff (Millwood) 25:4556, 2006

37. Washington CW, Derdeyn CP, Chicoine MR, Cross DT, Dacey RG, Moran CJ, et al: Comparing routine versus selective use of intraoperative cerebral angiography in aneurysm surgery: a prospective study. J Neurointerv Surg 8:75-80, 2016

38. Yoon PH, Kim DI, Jeon P, Lee SI, Lee SK, Kim SH: Pituitary adenomas: early postoperative MR imaging after transsphenoidal resection. AJNR Am J Neuroradiol 22:1097-1104, 2001

39. Ziu M, Dunn IF, Hess C, Fleseriu M, Bodach ME, Tumialan LM, et al: Congress of Neurological surgeons systematic review and evidence-based guideline on posttreatment follow-up evaluation of patients with nonfunctioning pituitary adenomas. Neurosurgery 79:E541-E543, 2016

\section{Disclosures}

Dr. Little has ownership interest in Kogent and Spiway. Dr. Yuen has received research grants to Barrow Neurological Institute from Pfizer, Novartis, Millendo, Ionis, Corcept, and Crinetics and has served on advisory boards for Novo Nordisk and Corcept.

\section{Author Contributions}

Conception and design: Alhilali, White. Acquisition of data: Alhilali, Lee, Ho. Analysis and interpretation of data: Alhilali, Little, Yuen, Lee, Fakhran, White. Drafting the article: Alhilali, Little, Yuen, Fakhran, White. Critically revising the article: Alhilali, Little, Yuen, Fakhran, White. Reviewed submitted version of manuscript: all authors. Approved the final version of the manuscript on behalf of all authors: Alhilali. Statistical analysis: Alhilali. Administrative/technical/material support: Alhilali. Study supervision: Alhilali, White.

\section{Correspondence}

Lea M. Alhilali: c/o Neuroscience Publications, Barrow Neurological Institute, St. Joseph's Hospital and Medical Center, Phoenix, AZ.neuropub@barrowneuro.org. 